\title{
NBS
}

Eechnical Note No.24 Boulder Laboratories

RADIO WAVE PROPAGATION IN AN INHOMOGENEOUS ATMOSPHERE

BY JAMES R. WAIT 


\section{THE NATIONAL BUREAU OF STANDARDS}

\section{Functions and Activities}

The functions of the National Bureau of Standards are set forth in the Act of Congress, March 3, 1901, as ainended by Congress in Public Law 619, 1950. These include the development and maintenance of the national standards of measurement and the provision of means and methods for making measurements consistent with these standards; the determination of physical constants ancl properties of inaterials; the development of methods and instruments for testing materials, devices. and structures; advisory services to government agencies on scientific and technical problems: invention and developinent of devices to serve special needs of the Government; and the development of standard practices, corles, and specifications. The work includes basic and applied research. development, engineering, instrumentation, testing, evaluation, calibration services, and various consultation and information services. Research projects are also performed for other government agencies when the work relates to and supplements the basic program of the Bureau or when the Bureau's unique competence is required. The scope of activities is suggested by the listing of divisions and sections on the inside of the back cover.

\section{Publications}

The results of the Bureau's work take the form of either actual equipment and devices or published papers. These papers appear either in the Bureau's own series of publications or in the journals of professional and scientific societies. The Bureau itself publishes three periodicals available from the Government Printing Office: The Journal of Research, published in four separate sections, presents complete scientific and technical papers; the Technical News Bulletin presents summary and preliminary reports on work in progress; and Basic Radio Propagation Predictions provides data for determining the best frequencies to use for radio communications throughout the world. There are also five series of nonperiodical publications: Monographs, Applied Mathematics Series, Handbooks, Miscellaneous Publications, and Technical Notes.

Information on the Bureau's publications can be found in NBS Circular 460, Publications of the National Bureau of Standards (\$1.25) and its Supplement (\$1.50), available from the Superintendent of Documents, Government Printing Office, Washington 25, D.C. 


\section{NATIONAL BUREAU OF STANDARDS Eechnical Mote}

\section{4}

September 10, 1959

RADIO WAVE PROPAGATION IN AN

INHOMOGENEOUS ATMOSPHERE

By

James R. Wait

This material was previously issued as a project report in October, 1958.

NBS Technical Notes are designed to supplement the Bureau's regular publications program. They provide a means for making available scientific data that are of transient or limited interest. Technical Notes may be listed or referred to in the open literature. They are for sale by the Office of Technical Services, U. S. Department of Commerce, Washington 25, D. C.

DISTRIBUTED BY

UNITED STATES DEPARTMENT OF COMMERCE

OFFICE OF TECHNICAL SERVICES

WASHINGTON 25, D. C.

Price $\$ 1.00$ 


\section{ABSTRACT}

A self-contained treatment of the theory of radio waves in an inhomogeneous atmosphere is given. The refractive index is assumed to vary with height above the earth in a monotonic fashion. Variation according to an exponential law is used for illustration of general principles. For this case, rigorous series formulas are developed for the distance to the horizon for an elevated point in the atmosphere. 



\section{ON RADIO WAVE PROPAGATION IN AN INHOMOGENEOUS ATMOSPHERE}

\section{Introduction and Formulation}

It is the purpose of this paper to review, and extend, work of Watson $^{1}$, Eckersley and Millington ${ }^{2}$, Bremmer $^{3}$, Vvedensky ${ }^{4}$ and Fock ${ }^{5}$ on propagation of radio waves in an inhomogeneous atmosphere. In particular an attempt will be made to present a unified treatment which includes the analyses of the above authors and shows that 'the distance to the horizon" is a valid concept in diffraction theory even when the atmosphere is not homogeneous. The earth is to be represented by a homogeneous sphere of radius a and the source is to be a vertical electric dipole which for the moment, is located near the earth's surface. The usual spherical coordinates $(r, \theta, \phi)$ are chosen with the source oriented along the polar axis and the surface of the earth is defined by $r=a$. The dielectric constant relative to free space is denoted by $\mathrm{K}$ and is understood to be a function of $\mathrm{r}$ and decreases monotonically with increasing $r$.

The resultant magnetic field has only a single component, $\mathrm{H}_{\phi}$, because of the assumed polar symmetry in the problem. As a further consequence of the symmetry, the electric field component $E_{\phi}$ is zero. It is convenient to express the remaining field components in terms of single scalar function $U$ which is actually the radial component of a Hertz vector. For a time factor exp (i $\omega t)$, this leads to

$$
\begin{gathered}
\mathrm{H}_{\phi}=i \mathrm{k}_{\mathrm{o}} \mathrm{K} \frac{\partial \mathrm{U}}{\partial \theta}, \\
\mathrm{E}_{\mathrm{r}}=\frac{120 \pi}{\mathrm{r} \sin \theta} \frac{\partial}{\partial \theta}\left(\sin \theta \frac{\partial \mathrm{U}}{\partial \theta}\right), \text { and } \\
\mathrm{E}_{\theta}=\frac{120 \pi}{\mathrm{Kr}} \frac{\partial}{\partial \mathrm{r}}\left(\mathrm{K} r \frac{\partial \mathrm{U}}{\partial \theta}\right)
\end{gathered}
$$

where $k_{0}=2 \pi /$ wavelength in vacuo. 
These field components satisfy Maxwell's equations if the function $U$ satisfies

$$
\frac{\partial}{\partial r}\left[\frac{1}{K} \frac{\partial}{\partial r}(K r U)\right]+\frac{1}{r \sin \theta} \frac{\partial}{\partial \theta}\left[\sin \theta \frac{\partial U}{\partial \theta}\right]+k_{0}^{2} K r U=0
$$

Following the work of Fock ${ }^{5}$, it now proves to be convenient to introduce an auxiliary quantity $U^{\prime}$ which is defined by

$$
U^{\prime}=e^{-i k a \theta} \mathrm{Kr} \sqrt{\sin \theta} \mathrm{U}
$$

and consequently the equation for $U$ satisfies

$$
\begin{aligned}
\frac{\partial^{2} U^{\prime}}{\partial r^{2}} & -\frac{2 i k}{a} \frac{\partial U^{\prime}}{\partial \theta}+k^{2}\left(\frac{K}{K_{0}}-\frac{a^{2}}{r^{2}}\right) U^{\prime} \\
& =\frac{K^{\prime}}{K} \frac{\partial U^{\prime}}{\partial r}-\frac{1}{r^{2}}\left[\frac{\partial^{2} U^{\prime}}{\partial \theta^{2}}+\left(\frac{1}{4}+\frac{1}{r \sin ^{2} \theta}\right) U^{\prime}\right]
\end{aligned}
$$

where $K^{\prime}=d K / d h, K_{0}$ is the value of $K$ at $r=a$, and $k=k_{0} \sqrt{K_{0}}$ is the effective wave number at the surface of the earth.

Since $E_{\theta} \cong-\mathrm{ZH}_{\phi}$ at $\mathrm{r}=\mathrm{a}$ it follows that

$$
\frac{\partial U^{\prime}}{\partial r}=i k\left(K_{0}\right)^{\frac{1}{2}} Z^{\prime} \text { at } r=a
$$

where $Z$ is the effective value of the surface impedance ${ }^{6-8}$.

The right-hand side of equation (6) can be replaced by zero. The justification for doing this is based on the fact that

$$
\partial U^{\prime} / \partial r=\text { Order of }\left(k U^{\prime} / M\right)
$$

and

$$
\partial U^{\prime} / \partial \theta=\text { Order of }\left(\mathrm{kaU} / \mathrm{M}^{2}\right)
$$

where $M=(\mathrm{ka} / 2)^{1 / 3}$ is a large quantity. It is also possible to simplify equation (6) by noting that

$$
\left(1-a^{2} / r^{2}\right) \cong 2 h / a \text {. }
$$


Consequently $U^{\prime}$ satisfies

$\frac{\partial^{2} U^{\prime}}{\partial h^{2}}-2 i k \frac{\partial U^{\prime}}{\partial s}+k^{2}\left(\frac{K-K_{0}}{K_{0}}+\frac{2 h}{2}\right) U^{\prime}=0$

where $s=a \theta$ is the great-circle distance measured along the earth's surface. The boundary condition at the surface of the earth then

becomes

$$
\partial U^{\prime} / \partial h=+i k\left(K_{0}\right)^{\frac{1}{2}} Z^{\prime} U^{\prime}(a t h=0) .
$$

The function $U^{\prime}$, which must satisfy equations (8) and (9).

should also have the proper singularity at the source. That is it should behave as

$$
U=\frac{e^{-i k R}}{R}\left(I+R_{v}\right)
$$

near the source, where $R_{v}$ is a Fresnel reflection coefficient of the ground for vertically polarized waves. For $h<<s$, and assuming that our approximate boundary condition is valid, $R \cong s+\left(h^{2} / 2 s\right)$

and thus

$$
\underset{\mathrm{v}}{\mathrm{R}} \cong \frac{\mathrm{h} / \mathrm{s}-\mathrm{Z}}{\mathrm{h} / \mathrm{s}+\mathrm{Z}}
$$

Consequently, the required behavior of U' near the origin is

$$
U^{\prime} \cong K_{0}(a / s)^{\frac{1}{2}}(2 h / s)(h / s+z)^{-1} e^{-i \frac{k h^{2}}{2 s}}
$$

\section{Equivalent Earth Radius Concept}

The equivalent earth radius a will now be defined in the following manner

$$
\frac{1}{a_{e}}=\frac{1}{a}+\frac{K_{0}^{\prime}}{2 K_{0}} \text { where } K_{o}^{p}=\left(\frac{\partial K}{\partial h}\right)_{h=0}
$$

This equation is usually justified on the basis of geometrical optics. It can also be demonstrated in a more rigorous fashion by rewriting equation ( 8 ) in the form

$$
\frac{\partial^{2} U^{\prime}}{\partial h^{2}}-2 i k \frac{\partial U^{\prime}}{\partial s}+\frac{2 k^{2}}{a_{e}} h(1+g) U^{\prime}=0
$$


where

$$
g=\frac{{ }_{e}}{2 K_{0}}\left(\frac{K-K_{0}}{h}-K_{0}^{\prime}\right) \text {. }
$$

Now if the atmosphere is linear then by definition $K_{0}^{\prime}=\left(K-K_{0}\right) / h$ and $y=0$ and consequently

$$
\frac{\partial^{2} U^{\prime}}{\partial h^{2}}-2 i k \frac{\partial U^{\prime}}{\partial s}+\frac{2 k^{2}}{a_{e}} h U^{\prime}=0 \text {. }
$$

But, on the other hand, if $K=K_{0}$ for all $h$ then from equation (8) or (14),

$$
\frac{\partial^{2} U^{\prime}}{\partial h^{2}}-2 i k \frac{\partial U^{\prime}}{\partial s}+\frac{2 k^{2}}{a} h U^{\prime}=0 .
$$

This strongly suggests that propagation in a linear atmosphere of radius a is equivalent to propagation over an earth of modified radius $a_{e}$ with a homogeneous atmosphere. It is therefore concluded that the effective earth radius is indeed a valid concept. This conclusion has also been reached by many other authors, including Bremmer, ${ }^{3}$ Fock ${ }^{5}$ and Miller ${ }^{9}$.

\section{Extension to Non-Linear Atmosphere}

In many practical cases of interest, howeve $r, K$ is not a linear function of height, particularly at great heights. Then the function $g$ is non-zero and is itself, in general, a function of height. To further analyze this case, the revelant equations will be put in dimensionless form. The following parameters are first introduced

$$
\begin{aligned}
& x=\left(\frac{k a_{e}}{2}\right)^{\frac{1}{3}} \frac{s}{a_{e}}=\left(\frac{k a_{e}}{2}\right)^{\frac{l}{3}} \theta, \\
& y=\left(\frac{2}{k a_{e}}\right)^{\frac{1}{3}} k h, \\
& q=-i\left(\frac{k a_{e}}{2}\right)^{\frac{1}{3}}\left(K_{o}\right)^{\frac{l}{2}}(Z / 120 \pi), \text { and } \\
& w=\frac{1}{K_{o}}\left(\frac{2}{k a_{e}}\right)^{1 / 6} U^{r} .
\end{aligned}
$$


The equation for $W$ is

$$
\frac{\partial^{2} w}{\partial y^{2}}-i \frac{\partial w}{\partial x}+y(1+g) w=0
$$

and the boundary condition at the earth's surface now becomes

$$
\frac{\partial w}{\partial y}+q w=0 \text { at } y=0 \text {. }
$$

Furthermore, as $x$ tends to zero, $W$ should behave as $(2 / \sqrt{x})$. $\exp \left(-i y^{2} / 4 x\right)$

It is now desirable to first consider the solution of equation (17) when y can be neglected compared to unity. This corresponds to an atmosphere which is linear or near linear. The revelant equation is now

$$
\frac{\partial^{2} w}{\partial y^{2}}-i \frac{\partial w}{\partial x}+y w=0
$$

This can be solved by the method of separation of variables. That is, as suming

$$
W=X(x) Y(y)
$$

one finds

$$
\frac{1}{Y(y)} \frac{d^{2} Y(y)}{d y^{2}}=i \frac{1}{X(x)} \frac{d X(x)}{d x}=t
$$

where $t$ the separation parameter. Thus

and

$$
\frac{d X}{d x}+\text { it } X=0
$$

$$
\frac{d^{2} Y}{d t^{2}}+(y-t) Y=0
$$

Solutions of the first equation are

$$
X(x)=e^{ \pm i t x}
$$

and the second equation has solutions of the form

$$
Y(y)=W(t-y)
$$

where $W(t)$ satisfies Airy's equation

$$
\frac{d^{2} W(t)}{d t^{2}}-t w(t)=0 \text {. }
$$


The appropriate solution for the $\mathrm{x}$ dependence is chosen such that outgoing waves are only retained. Therefore

$$
X(x)=e^{-i t x} \quad \text {. }
$$

Furthermore, the radial part of the solution (i.e. y dependence) is chosen so that the phase of $\mathrm{Y}(\mathrm{y})$ is a decreasing function with height (i.e. phase lag increases with height). At this stage, it should be noted that Airy's equation is closely related to Bessel's equation of order $1 / 3$. In fact

$$
w(t)=\text { const. } \times t^{\frac{1}{2}} z_{1 / 3}\left[(2 / 3)(-t)^{2 / 3}\right]
$$

where $\mathrm{Z}_{1 / 3}$ is any cylindrical Bessel function or linear combinations thereof. In order to satisfy our requirement that the phase lag be an increasing function with height, the Hankel function $\mathrm{H}_{1}^{(2)} / 3$ of the second must be used. A particular solution which is suitable from the above standpoint is

$$
W(t)=W_{1}(t)=e^{-i 2 \pi / 3}(-\pi t / 3)^{\frac{1}{2}} H_{1 / 3}^{(2)}\left[(2 / 3)(-t)^{3 / 2}\right]
$$

where the constant is chosen to be consistent with the work of Fock and Leontovich. In fact, the latter authors represent $W_{1}(t)$ as an integral of the Airy type

$$
w_{1}(t)=\frac{1}{\sqrt{\pi}} \int_{\infty}^{\infty} i 2 \pi / 3 e^{t z-z^{3 / 3}} d z
$$

where the contour is a straight line from infinity along the straight arg. $t=2 \pi / 3$ to the origin and then out to $\infty$ along the real axis. (i.e. arg. $t=0$ ).

To satisfy the boundary condition at the earth's surface, one must choose

$$
\left[w_{1}^{\prime}(t-y)-q w_{1}(t-y)\right]_{y=0}=0 .
$$

A numerical study of this equation indicates that the roots $t=t_{S}$ $(s=0,1,2 \ldots)$ are in the fourth quadrant of the $t$ plane. The solution is then made up a linear combination of terms of the type $e^{-i x t_{s}} w_{1}\left(t_{s}-y\right)$. 
It can be seen immediately that an equivalent representation is

$$
w_{1}=\gamma \int_{C} \frac{e^{-i x t} w_{1}(t-y)}{w_{1}^{\prime}(t)-q w_{1}(t)} d t
$$

where $C$ is some contour which encloses the poles of the integrand (i.e. at $t=t_{s}$ ). The factor $\gamma$, assumed constant, is to be chosen such that $W_{1}$ has the proper singularity as $x$ approaches zero. It is to be expected that behavior of $W_{1}$ for small $x$ is to be determined by the behavior of the integrand for large values of $t$ because of the factor $e^{-i x t}$. But, when $t$ is very large, it follows from the known asymptotic behavior of the Hankel functions, that

$$
\frac{W_{1}(t-y)}{W_{1}(t)-q W_{1}(t)} \cong \pm(t)^{-\frac{1}{2}} \exp \left[-y(t)^{\frac{1}{2}}\right]
$$

where the upper $(t)$ sign is to be employed for $2 \pi / 3 \geqq \arg t>-\pi / 3$ and the lower $(-)$ sign for $-\pi / 3>\arg t \geqq 4 \pi / 3$. The contour $C$ is now drawn such that one branch is along the negative imaginary axis $(-i \infty$ to 0$)$ and the other along the real axis $(0$ to $\infty)$. For small values of $x$, then

$$
\begin{aligned}
& w \sim \gamma \int_{0}^{-i \infty} e^{-i x t} \frac{e^{y \sqrt{t}}}{\sqrt{t}} d t \\
& \quad+\gamma \int_{0}^{\infty} e^{-i x t} \frac{e^{-y \sqrt{t}}}{\sqrt{t}} d t .
\end{aligned}
$$

These integrals can be transformed to the form

$$
\int_{0}^{\infty} e^{-\beta^{2}} d \beta=\sqrt{\pi} / 2
$$

and thus

$$
w \sim 2 y\left(\frac{\pi}{i x}\right)^{\frac{1}{2}} e^{-i \frac{y^{2}}{4 x}}
$$

and therefore, if $\gamma=(i / \pi)^{\frac{1}{2}}$ 


$$
W \cong 2 x^{-\frac{1}{2}} e^{-1 \frac{y^{2}}{4 x}}
$$

as $\mathrm{x}$ approaches zero.

The required solution can thus be written

$$
W=\left(\frac{i}{\pi}\right)^{\frac{1}{2}} \int_{C} \frac{e^{-i x t} W_{1}(t-y)}{W_{1}(t)-q W_{1}(t)} d t .
$$

In this particular case the solution for $U$ becomes

where $V=x^{\frac{1}{2}} w$

$$
U=\frac{e^{-i k a \theta}}{a(\theta \sin \theta)^{\frac{1}{2}}} V \cong \frac{e^{-i k a \theta}}{a \theta} V
$$

$$
V=\left(\frac{i x}{\pi}\right)^{\frac{1}{2}} \int_{C} \frac{e^{-i x t} W_{1}(t-y)}{W_{1}(t)-q W_{1}(t)} d t .
$$

Attention is now returned to the case of an inhomogeneous atmosphere wherein $g(y)$ is no longer negligible compared to unity. The more general equation for $W$ must then be retained

$$
\frac{\partial^{2} W}{\partial y^{2}}-i \frac{\partial w}{\partial x}+y[1+g(y)] w=0 \text {. }
$$

Proceeding in an analogous fashion as in the linear case, it is readily found that solutions are of the form

$$
W=e^{-i x t} F(y, t)
$$

where $F(y, t)$ must satisfy

$$
\frac{d^{2} F(y, t)}{d y^{2}}+[y-t+y g(y)] F(y, t)=0 .
$$

The solution is then expressed as an integral

$$
w_{1}=\sqrt{\frac{i}{\pi}} \int \frac{e^{-i x t} F(y, t)}{\left[\frac{\partial F}{\partial y}+q F\right]_{y=0}} d t
$$


which is constructed so the poles of the integrand are the roots, $t=t_{s}$, of the equation

$$
\left[\frac{\partial F(y, t)}{\partial y}+q F(y, t)\right]_{y=0}=0
$$

and the constant term outside the integral is chosen so that it reduces to the corresponding expression for $W_{1}$ in the linear atmosphere (i.e. $g=0$ ). The corresponding (formal) solution for the function $U$ is thus written

$$
U=\frac{e^{-i k a \theta}}{a(\theta \sin \theta)^{\frac{1}{2}}} \mathrm{~V}
$$

where

$$
V=\left(\frac{i x}{\pi}\right)^{\frac{1}{2}} \int_{C} \frac{e^{-i x t} F(y, t)}{\left[\frac{\partial F}{\partial y}+q F\right]_{y=0}} d t \text {. }
$$

\section{Asymptotic Form of the Solution}

Various asymptotic approximations are now investigated. For large values of the real part of $(y-t+y g)$, the function $F(y, t)$ of equation (40) is given approximately by the W.K.B. solution of equation (40). This is

$$
F(y, t) \cong \frac{C e^{-i \pi / 4}}{[y-t+y g(y)]^{\frac{1}{4}}} \exp \left[-i \int_{t}^{y}[u-t+u g(u)]^{\frac{1}{2}} d u\right]
$$

where $\mathrm{C}$ is a constant and the lower limit of the integral is arbitrary. It should be noticed that if $\mathrm{g}=0$

$$
F(y, t)=C w_{1}(t-y)
$$

which for large $t-y$ behaves asymptotically like

$$
F(y, t) \cong \frac{C e^{-i \pi / 4}}{(y-t)^{\frac{1}{4}}} \exp \left[-i \int_{t}^{y}(u-t)^{\frac{1}{2}} d u\right]
$$

in accordance with equation (28).

Equation (46) for $\mathrm{V}$ in the case of an inhomogeneous atmosphere can now be simplified by noting $F(y, t)$, where it occurs in the denominator, can be replaced by $W_{1}(t-y)$ and therefore $\left(\frac{\partial F}{\partial y}+q F\right)_{y=0}=-w_{1}^{\prime}(t)+q W_{1}(t)$. 
In the numerator $F$ can be replaced by the asymptotic form given by equation (46) where y is large. Thus

$V=-\frac{1}{2}\left(\frac{x}{i \pi}\right)^{\frac{1}{2}} \int_{C}\left[\frac{1}{y-t+y g(y)}\right]^{\frac{1}{4}} \frac{e^{-i \Omega}}{w_{1}^{1}(t)-q W_{1}(t)} d t$

whe re

$$
\Omega=x t+\int_{0}^{y} \sqrt{u-t+u g(u)} d u .
$$

The saddle point, determined by $d \Omega / d t=0$, is thus given by $t=-p^{2}$ where

$$
\int_{0}^{y} \frac{d u}{\sqrt{u+p^{2}+u g(u)}}-2 x=0 \text {. }
$$

Thus, following the prescription of the saddle point method

$$
v \cong e^{-i \Omega_{0}}\left(2 x \frac{\partial p}{\partial y}\right)^{\frac{1}{2}} F(-p)
$$

where

$$
F(-p)=\frac{1}{\sqrt{\pi}} \int_{C} e^{i p t} \frac{d t}{W_{1}(t)-q W_{1}(t)}
$$

and

$$
\Omega_{0}=-x^{2}+\int_{0}^{y}\left[u+p^{2}+u g(u)\right]^{\frac{1}{2}} d u .
$$

The integral $F(-p)$ has been discussed on previous occasions and numerical results are available ${ }^{10}$. For large negative values of the argument (i.e. p $>0$ ) it was noted that

$$
F(-p) \cong \frac{2 p}{p+i q} \exp \cdot\left(-i p^{3} / 3\right)
$$

and therefore

$$
v \cong e^{-i \Omega_{0}}\left(2 x \frac{\partial p}{\partial y}\right)^{\frac{1}{2}} \frac{2 p}{p+i q} \exp \cdot\left(-i p^{3} / 3\right)
$$

must be the geometrical optical approximation and consequently, the factor $2 p /(p+i q)$ can be identified as $1+R(a)$ where $R(a)$ is the Fresnel reflection coefficient for a plane wave incident at an angle $a$ on the boundary. The parameter $\mathrm{p}$ is then related to $a$ by 


$$
\mathrm{p}=\left(\mathrm{ka}_{\mathrm{e}} / 2\right)^{\frac{1}{3}} \cos a \text {. }
$$

In the case of the linear atmosphere (i.e. $g(u)=0$ )

$$
\begin{aligned}
\Omega_{0} & =-x p^{2}+\int_{0}^{y}\left(u+p^{2}\right)^{\frac{1}{2}} d u \\
& \cong p^{3} / 3+2 y^{3 / 2} / 3
\end{aligned}
$$

for $p$ small compared to $x$ and $y$. In this same case

$$
p \cong \frac{y-x^{2}}{2 x}
$$

and consequently

$$
\left(2 x \frac{\partial p}{\partial y}\right)^{\frac{1}{2}} \cong 1 \text {. }
$$

When $g(u)=0$, the formulas thus reduce to those for a homogeneous atmosphere with a modified earth radius.

In the case of the inhomogeneous atmosphere equation (50) for $p$ can be solved approximately to yield

$$
p=\frac{1}{2} \int_{0}^{y} \frac{d u}{\sqrt{u+u g(u)}}-x
$$

under the condition that $x$ and $y$ are large but the difference $x-\sqrt{y}$ is finite. Thus

$$
\left(2 x \frac{\partial p}{\partial y}\right)^{\frac{1}{2}} \cong \frac{x^{\frac{1}{2}}}{[y+y g(y)]^{\frac{1}{4}}} \cong 1
$$

and

$$
\Omega_{0} \cong \mathrm{p}^{3} / 3+\int_{0}^{y}[u+u g(u)]^{\frac{1}{2}} d u .
$$

For application to an actual atmosphere, it is necessary to evaluate the integral

$$
p+x=\frac{1}{2} \int_{0}^{y} \frac{d u}{\sqrt{u+u g(u)}}
$$

where

$$
g=\frac{a_{e}}{2 K_{0}}\left(\frac{K-K_{0}}{h}-K_{0}^{\prime}\right)
$$

When the dielectric constant varies in an arbitrary, but montonic, way 
with height, the integral can be evaluated readily by numerical methods. Some simplicity is obtained if it can be assumed that the variation is exponential in form. For example if

$$
K-K_{0}=\left(K_{0}^{\prime} / c\right)\left(1-e^{-c h}\right)
$$

it is seen that $K$ decays exponentially from the value $K_{0}$ at the surface $(\mathrm{h}=0)$. The constant $\mathrm{c}$ is of the order 0.13 for an average atmosphere and $c$ measures of the rate of decay of the dielectric constant with increasing height. ${ }^{13}$ Before proceeding further in this direction, it is probably preferable to show the connection of the above results with those of geometrical optics.

\section{Distance to the Horizon}

The distance $s$ to the horizon from an elevated point in the atmosphere is often used conveniently as a parameter in comparing one type of vertical refractive index profile with another. Using a method based on a stationary phase principle, it readily follows from Bremme ${ }^{3}$ (and others) that

$$
s=n_{0} \int_{0}^{h} \frac{a^{2}}{r} \frac{d h}{\sqrt{[r n(r)]^{2}-n_{0} a^{2}}}
$$

where $r=a+h, n(r)$ is the refractive which is a function of $r$ and $n$ 。 is the surface value [i.e. $n_{0}=n(a)$ ].

In the case of exponential atmosphere

$$
\mathrm{n}=1+\mathrm{be} \mathrm{e}^{-\mathrm{ch}}=\sqrt{\mathrm{K}}
$$

so that

$$
\left.\mathrm{n}_{0}=\mathrm{n}\right]_{\mathrm{h}=0}=1,=\sqrt{\mathrm{K}}
$$

The term under the radical cai oe written

$$
\begin{aligned}
& a^{2}\left[\left(1+b e^{-c h}\right)^{2}\left(1+\frac{h}{a}\right)^{2}-(1+b)^{2}\right] \\
& \cong a^{2}\left[1+2 b e^{-c h}+2 \frac{h}{a}-1-2 b\right] \\
& \cong a^{2}\left[\frac{2 h}{a}+2 b\left(e^{-c h}-1\right)\right]
\end{aligned}
$$

under the assumption that $\mathrm{b}<<1$ and $\mathrm{h} / \mathrm{a}<<1$. To the same 
approximation $\frac{n_{0}}{r}$ in the integrand can be replaced by $\frac{1}{a}$. Consequently, the integral becomes

$$
s \cong \int_{0}^{h_{0}} \frac{d h}{\sqrt{(2 h / a)+2 b\left(e^{-c h}-1\right)}}
$$

which is equivalent to equation (61) derived from the full theory (with $p=0$ ). Now, it is convenient to break the integration into three parts, such that

$$
\int_{0}^{h_{0}}=\int_{0}^{h^{\prime}}+\int_{h^{\prime}}^{h^{\prime \prime}}+\int_{h^{\prime \prime}}^{h_{0}=h^{\prime \prime \prime}}
$$

and thus

$$
\mathrm{s}=\mathrm{s}^{\prime}+\mathrm{s}^{\prime \prime}+\mathrm{s}^{\prime \prime \prime} \text {. }
$$

In the first range $\left(0 \leqq h \leqq s^{\prime}\right)$

$$
s^{\prime} \cong \int_{0}^{h^{\prime}} \frac{d h}{\sqrt{(2 h / a)+2 b\left(-(c h)+\frac{(c h)^{2}}{2}\right)}}
$$

which is valid if $h^{\prime}$ is chosen such that the $(\mathrm{ch})^{3} / 6$ term in the expansion of $e^{-c h}$ is negligible compared to $c h$. This is satisfied if

$$
\left(\mathrm{ch}^{\prime}\right)^{2} / 6<<1
$$

(For example if, $\mathrm{ch}^{\prime}=\frac{1}{2}$ then $\left.\left(\mathrm{ch}^{1}\right)^{2} / 6=1 / 24\right)$.

Writing

$$
s^{\prime} \cong \frac{1}{\sqrt{(2 h / a)-2 l}} \int_{2}^{h^{\prime}} \frac{d h}{\sqrt{h(1+a h)}}
$$

we find

$$
\mathrm{s}^{\prime}=\frac{1}{\sqrt{2 / \mathrm{a}-2 \mathrm{bc}}} \overline{\sqrt{a}} \tanh ^{-1} \sqrt{\frac{a h^{\prime}}{1+a h^{\prime}}}
$$

where

$$
a=\frac{b c^{2}}{(2 / a)-2 b c} .
$$


If the effective earth's radius is defined by

$$
\frac{1}{a_{e}}=\frac{1}{a}+\frac{K_{0}}{2 K_{0}}
$$

where

$$
\mathrm{K}_{0}=(1+b)^{2} \cong 1+2 b
$$

and

$$
\begin{aligned}
K_{0}^{\prime} & =\left.\frac{d}{d h}\left(1+b e^{-c h}\right)^{2}\right|_{h=0} \\
& \cong-2 b c^{\prime}
\end{aligned}
$$

and thus

$$
\frac{1}{a_{e}}=\frac{1}{a}-b c
$$

so that

$$
s^{\prime}=\frac{1}{\sqrt{2 a / a_{e}}} \tanh ^{-1} \sqrt{\frac{a h^{\prime}}{1+a h}}
$$

where

$$
a=\frac{c}{\sqrt{2 / a_{e}}}\left(\frac{1}{a}-\frac{1}{a_{e}}\right)
$$

if $a h^{\prime}<<1$

$$
s^{\prime} \cong \frac{1}{\sqrt{2 a / a} e} \sqrt{a h^{\prime}}=\sqrt{2 a e^{h^{\prime}}}
$$

which is the usual approximation for the distance to the horizon from a height $h^{\prime}$ in a linear atmosphere. The hyperbolic tangent formula above is actually the distance to the horizon from a height $h^{\prime}$ in an atmosphere defined by

$$
\mathrm{n}=1+\mathrm{b}\left(1-\mathrm{ch}+(\mathrm{ch})^{2} / 2\right)
$$

for $0 \leqq h \leqq h^{\prime}$. This is an approximation to

$$
\mathrm{n}=1+\mathrm{be}^{-\mathrm{ch}}
$$

as mentioned above.

We shall turn our attention to the third portion (i. e. $\left.h^{\prime \prime} \leqq h \leqq h^{\prime \prime \prime}=h_{0}\right)$;

$$
s^{\prime \prime \prime}=\int_{h^{\prime \prime}}^{h_{0}} \frac{d h}{\sqrt{(2 h / a)+2 b\left(e^{-c h}-1\right)}} \text {. }
$$


Now the term in the radical is written in the following form

$$
(2 h / a-2 b)+2 b e^{-c h}=(2 h / a-2 b)\left[1+\frac{2 b e^{-c h}}{2 h / a-2 b}\right] \text {. }
$$

Letting $u=(2 \mathrm{~h} / \mathrm{a}-2 \mathrm{~b})$ it follows that

$$
\begin{gathered}
\frac{1}{\left[u\left(1+\frac{2 b c^{-c h}}{u}\right)\right]^{\frac{1}{2}}}=\frac{1}{\sqrt{u}} \sum_{n=0,1,2,3 \ldots A_{n} \frac{(2 b)^{n}}{u^{n}} e^{-c h n}} \\
=\sum_{n} A_{n}(2 b)^{n} e^{-c n b a} \times\left[\frac{e^{-\frac{c n a}{2} u}}{u^{n+1 / 2}}\right]
\end{gathered}
$$

where $A_{0}=1, A_{1}=-\frac{1}{2}, A_{2}=\frac{1.3}{2.4}$

$$
A_{3}=-\frac{1 \cdot 3 \cdot 5}{2 \cdot 4 \cdot 6}, A_{4}=\frac{1 \cdot 3 \cdot 5 \cdot 7}{2 \cdot 4 \cdot 6 \cdot 8} \text { and so on }
$$

provided $\frac{2 b e^{-c h}}{\mathrm{u}}<1$. This is satisfied if $2 \mathrm{~b}<\mathrm{h} / \mathrm{a} \leqq \mathrm{h}^{\prime \prime} / \mathrm{a}$ or if $h^{\prime \prime}>2 \mathrm{ba} \cong 2 \times 3 \times 10^{-4} \times 6360 \cong 3.9 \mathrm{~km}$ for any value of $\mathrm{c}$. Actually, this is more stringent than necessary since $e^{-c h}<1$. Thus

$$
s^{\prime \prime \prime}=\sum_{n=0,1,2 \ldots} A_{n}(2 b)^{n} e^{-c n b a} \int_{u^{\prime \prime}}^{u_{0}} \frac{e^{-\frac{c n a}{2} u}}{u^{n+1 / 2}} d u(a / 2) .
$$

Now consider the integral which occurs in the above expression,

$$
v_{m}(\beta u)=\int_{u}^{\infty} \frac{e^{-\beta u}}{u^{m+1 / 2}} d u
$$

and integrate it by parts successively to yield the recurrence relations

$$
\begin{aligned}
& v_{m}(\beta u)=\frac{1}{(m-1 / 2)}\left[\frac{e^{-\beta u}}{u^{m-1 / 2}}-\beta v_{m-1}(\beta u)\right] \\
& v_{m-1}(\beta u)=\frac{1}{(m-3 / 2)}\left[\frac{e^{-\beta u}}{u^{m-3 / 2}}-\beta v_{m-2}(\beta u)\right] \\
& \ldots-\ldots-\ldots \text { and so on, until }
\end{aligned}
$$




$$
v_{2}(\beta u)=\frac{1}{(1 / 2)}\left[\frac{e^{-\beta u}}{u^{3 / 2}}-\beta v_{1}(\beta u)\right]
$$

where

$$
v_{1}(\beta u)=\int_{u}^{\infty} \frac{e^{-\beta u}}{u / 2} d u=\left(\frac{\pi}{\beta}\right)^{\frac{1}{2}} \operatorname{erfc}(\sqrt{\beta u})
$$

where

$$
\operatorname{erfc}(\sqrt{\beta u})=\frac{2}{\sqrt{\pi}} \int_{\sqrt{\beta u}}^{\infty} e^{-x^{2}} d x
$$

is the complement of the error integral which is extensively tabulated. 14 Finally

$$
s^{\prime \prime}=\frac{a}{2} \sum_{n} A_{n}(2 b)^{n} e^{-n b c a}\left[v_{n}\left(\beta u^{\prime \prime}\right)-v_{n}\left(\beta u_{0}\right)\right]
$$

where

$$
\beta=\frac{c n a}{2}, u^{\prime \prime}=2\left(\frac{h^{\prime \prime}}{a}-b\right)
$$

and

$$
u_{0}=2\left(\frac{h_{0}}{a}-b\right) \text {. }
$$

It would appear that in most cases $h^{\prime}$ can be taken equal to $h^{\prime \prime}$ and chosen so that $h^{\prime}=h^{\prime \prime}=4 \mathrm{~km}$ which is a convenient upper limit for the quadratic profile and a lower limit for the expansion formula. Thus $s=s^{1}+s^{\prime \prime}$.

An alternative is to return to the integral s' $\mathrm{s}^{\prime}$ and expand $\mathrm{e}^{-\mathrm{ch}}$ to terms of third order. This leads to

$$
s^{\prime \prime}=\frac{1}{\sqrt{(2 / a)-2 b c}} \int_{h^{\prime}}^{h^{\prime \prime}} \frac{d h}{\sqrt{h(1+\gamma h)(1+\delta h)}}
$$

where

$$
\gamma=\frac{b c^{2}}{2\left(\frac{1}{a}-b c\right)} \text { and } \delta=\frac{b c}{6\left(\frac{1}{a}-b c\right)} \text {. }
$$

This integral is expressible in terms of elliptic integrals (see Pierce ${ }^{14}$ No.535 for example). 


\section{Concluding Remarks}

The present analysis provides a strong justification for the validity of an equivalent earth radius concept when the refractive index of the atmosphere varies in a linear manner with height in agreement with the conclusions of Bremmer ${ }^{3}$, Norton ${ }^{11}$, Miller ${ }^{9}$ and Northover ${ }^{16}$. The effect of the non-linearity becomes important at great heights as pointed out by Millington ${ }^{15}$ and others ${ }^{10,17}$ who have suggested that the theory for a linear atmosphere could be modified by simply relocating the optical horizon point. In the present paper a complete mathematical justification is given for this step provided that the variation of the refractive index with height is monotonic.

The idea that the structure of the diffraction fields in a monotonically varying or tapered atmosphere is similar to that for a homogeneous or airless atmosphere is in apparent contradiction to Carroll's theory ${ }^{18}$ of "radio twilight". However, the model he uses is not smoothly tapered since there are discontinuities in the gradients of the refractive index. In the bilinear mode1, for example, the atmosphere is represented by a layer of dielectric with a constant gradient up to about 30,000 feet after which the gradient is zero. Carroll obtains his solution by matching the appropriate wave functions at the atmosphere-free space interface ${ }^{19}$. In the free space region (above 30,000 feet for the bilinear model) only outgoing waves are allowed but at the top of the dielectric graded layer the two types of wave functions which are used can be identified as upgoing and downcoming waves. In the present treatment which uses a modified W.K.B. method, the solution in this region does not have this double character.

Other models used by Carroll and Ring ${ }^{19,20}$ such as the inversesquare and trilinear profiles can be objected to on similar grounds. It is of interest to note that the inverse-square profile is somewhat better in that the discontinuity is in the second derivative at the bounding surface and the computed attenuation, as expected, is greater. The trilinear model has two piece-wise linear sections with interfaces at 15,000 and 90,000 feet and the mode computations show less attenuation 
which is probably a consequence of the strong reflections from the lower interface.

It is the opinion of this writer that the Carroll and Ring computations are correct for the models specified. However, they cannot be regarded as valid solutions for propagation in a monotonically tapered layer. It is probable if the model were chosen so that the higher-order derivatives of the refractive index are continuous at the interfaces, the mode solutions and the modified W.K.B. solutions should approach one another. Unfortunately, the poor convergence of the resulting mode solutions will make this ultimate comparison very difficult. 


\section{References}

1. G. N. Watson, "The Diffraction of Radio Waves by the Earth", Proc.Royal Society London, V. A95, pp. 83-99, 1918, V. A95, p. 546-563, 1919.

2. G. Millington, "The Diffraction of Wireless Waves Round the Earth", (A summary of the diffraction analysis, with a comparison between the various methods), Phil. Mag., S. 7, V. 27, No. 184, pp.517-542, May, 1939, and T. L. Eckersley and G. Millington, Phil. Trans. Roy. Soc. No. 778, Vol. 237, pp. 273-309, 1938.

3. B. Van der Pol, H. Bremmer, "The Diffraction of Electromagnetic Waves from an Electrical Point Source round a Finitely Conducting Sphere, with Applications to Radiotelegraphy and the Theory of the Rainbow; The Propagation of Radio Waves over a Finitely Conducting Spherical Earth", Phil. Mag. S. 7, Vol. 24, No. 159, pp. 141-176, July, 1937, S. 7, V. 24, No. 164, pp. 825-864, Suppl., November 1947 , S. 7, V. 25, No. 171, pp. 817-834, Suppl., June, 1938, S. 7, V. 27, No. 182, pp. 261-275, March, 1939, and H. Bremmer, "Terrestrial Radio Waves", Elsevier, New York, 1949.

4. B. Vvedensky, "The Diffractive Propagation of Radio Waves", Techn. Phys. U.S.S.R., V. 2, pp. 624-639, 1935, V. 3, pp.915-925, 1936, V. 4, pp. 579-591, 1937.

5. V. A. Fock, "Diffraction of Radio Waves Around the Earth's Surface", Jour.Phys. U.S.S.R., Vol. 9, pp. 256-266, 1945, Jour. Theo. Exp. Phys., Vol. 15, pp. 480-490, 1945 and M. A. Leontovich and V. A. Fock, "Propagation of Electromagnetic Waves Along the Earth's Surface", Jour. Phys., U.S.S.R., Vol. 10, pp. 13-24, 1946.

6. M. A. Leontovich, "Approximate Boundary Conditions for the Electromagnetic Field on the Surface of a Good Conductor", Bull. Academy Sciences, U.S.S.R. serie physique, Vol. 9, p. 16, 1944. (in Russian).

7. G. D. Monteath, "Application of the Compensation Theorem to Certain Radiation and Propagation Problems", Proc.Inst. Elect. Engrs. Vol. 98, Pt. IV, p. 23-30, 1951.

8. J. R. Wait and W. J. Surtees. "Impedance of a Top-Loaded Antenna of A rbitrary Length over a Circular Grounded Screen", Jour. App. Physics, Vol. 25, No.5, pp. 553-555, May, 1954.

9. W. E. Miller, "Effective Earth's Radius for Radio Wave Propagation Beyond the Horizon", Jour. App. Phys.Vol.22, pp.55-62, Jan., 1952.

10. J. R. Wait and A. M. Conda, "Radiation Pattern of an Antenna on a Curved Lossy Surface", Trans. I. R.E., Vol. AP-6, pp. 348-359, October, 1958. 
11. K. A. Norton, "The Calculation of Ground-Wave Field Intensity Over a Finitely Conducting Spherical Earth", P.I. R.E., V. 29 pp. 623-639, December, 1941, and "Transmission Loss in Radiowave Propagation", Pt. II to be published.

12. L. J. Anderson, "Tropospheric Bending of Radio Waves", Trans. AGU Vol. 39, No.2, pp. 208-212, April, 1958.

13. B. R. Bean and G. D. Thayer, "On Models of the Atmospheric Radio Refractive Index", to be published in Proc. I. R. E. (Federal Govt. Issue, 1959).

14. B. O. Pierce, "A Short Table of Integrals", Ginn and Co., 3 rd Rev. Ed. 1929.

15. G. Millington, "Propagation at Great Heights in the Atmosphere"; The Marconi-Review, Vol. 21, pp. 143-159, 1958.

16. F. H. Northover, "Long Distance V.H.F. Fields", Can. Jour. Phys. Vol. 33, pp.241-256, May, 1955.

17. Personal communications from K. A. Norton, J. W. Herbstreit and $H$. Bremmer.

18. T. J. Carroll, "Internal Reflection in the Troposphere and Propagation well beyond the Horizon", Trans. P.G.A.P. Vol. 4, p. 19, Dec., 1952.

19. T. J. Carroll and R. M. Ring, "Propagation of Short Radio Waves in a Normally Stratified Troposphere", Proc. I. R. E., Vol. 43. pp. 1384-1390, Oct., 1955 .

20. T. J. Carroll and R. M. Ring, "Twilight Region Propagation of Microwaves by a Monotonically Tapered Layer of Air Dielectric", L'onde Electrique Vol. 37, pp. 471-479, May, 1957 (in French). 


\section{THE NATIONAL BUREAU OF STANDARDS}

The scope of activities of the National Bureau of Standards at its major laboratories in Washington, D.C., and Boulder, Colorado, is suggested in the following listing of the divisions and sections engaged in technical work. In general, each section carries out specialized research, development, and engineering in the field indicated by its title. A brief description of the activities, and of the resultant publications, appears on the inside of the front cover.

\section{WASHINGTON, D.C.}

Electricity and Electronics. Resistance and Reactance. Electron Devices. Electrical Instruments. Magnetic Measurements. Dielcctrics. Engineering Electronics. Electronic Instrumentation. Electrochemistry.

Optics and Metrology. Photometry and Colorimetry. Photographic Technology. Length. Engineering Metrology.

Heat. Temperature Physics. Thermodynamics. Cryogenic Physics. Rheology. Molecular Kinetics. Free Radicals Research.

Atomic and Radiation Physics. Spectroscopy. Radiometry. Mass Spectrometry. Solid State Physics. Electron Physics. Atomic Physics. Neutron Physics. Radiation Theory. Radioactivity. X-rays. High Energy Radiation. Nucleonic Instrumentation. Radiological Equipment.

Chemistry. Organic Coatings. Surface Chemistry. Organic Chemistry. Analytical Chemistry. Inorganic Chemistry. Electrodeposition. Molecular Structure and Properties of Gases. Physical Chemistry. Thermochemistry. Spectrochemistry. Pure Substances.

Mechanics. Sound. Mechanical Instruments. Fluid Mechanics. Engineering Mechanics. Mass and Scale. Capacity, Density, and Fluid Meters. Combustion Controls.

Organic and Fibrous Materials. Rubber. Textiles. Paper. Leather. Testing and Specifications. Polymer Structure. Plastics. Dental Research.

Metallurgy. Thermal Metallurgy. Chemical Metallurgy. Mechanical Metallurgy. Corrosion. Metal Physics.

Mineral Products. Engineering Ceramics. Glass. Refractories. Enameled Metals. Constitution and Microstructure.

Building Technology. Structural Engineering. Fire Protection. Air Conditioning, Heating, and Refrigera tion. Floor, Roof, and Wall Coverings. Codes and Safety Standards. Heat Transfer. Concreting Materials.

Applied Mathematics. Numerical Analysis. Computation. Statistical Engineering. Mathematical Physics.

Data Processing Systems. SEAC Engineering Group. Components and Techniques. Digital Circuitry. Digital Systems. Analog Systems. Application Engineering.

- Office of Basic Instrumentation.

- Office of Weights and Measures.

\section{BOULDER, COLORADO}

Cryogenic Engineering. Cryogenic Equipment. Cryogenic Processes. Properties of Materials. Gas Liquefaction.

Radio Propagation Physics. Upper Atmosphere Research. Ionospheric Research. Regular Propagation Services. Sun-Earth Relationships. VHF Rcsearch. Radio Warning Services. Airglow and Aurora. Radio Astronomy and Arctic Propagation.

Radio Propagation Engineering. Data Reduction Instrumentation. Modulation Research. Radio Noise. Tropospheric Measurements. Tropospheric Analysis. Propagation Obstacles Engineering. Radio-Meteorology. Lower Atmosphere Physics.

Radio Standards. High Frcquency Electrical Standards. Radio Broadcast Service. High Frcquency Inı. pedance Standards. Electronic Calihration Center. Microwave Physics. Microwave Circuit Standards.

Radio Communication and Systems. Low Frequency and Very Low Frcquency Research. High lirequcncy and Very lligh Frequency Research. Ultra High Frcquency and Super lligh Frequeney Rcsearch. Modulation Research. Antenna Research. Navigation Systems. Systcms Analysis. Field Operations. 

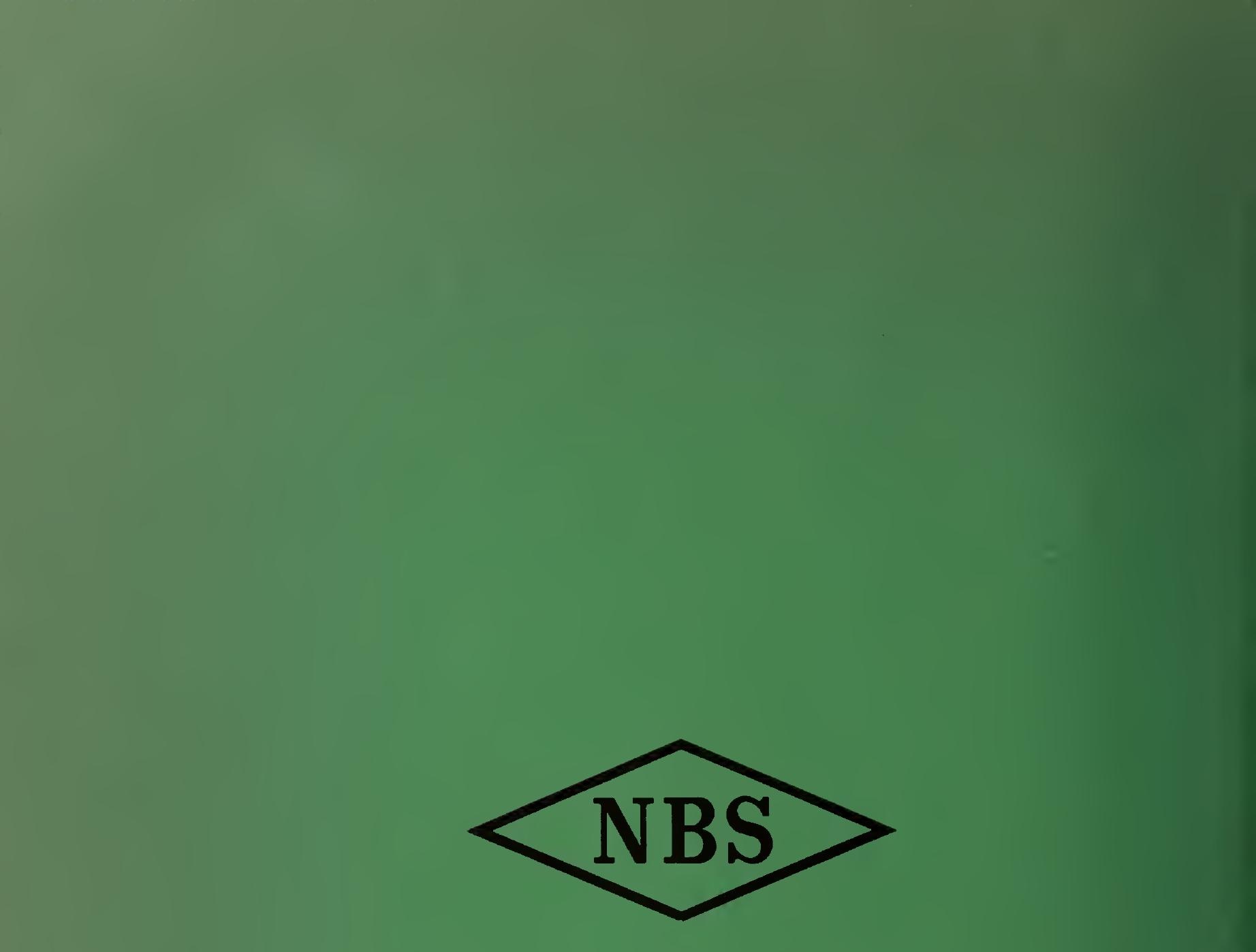